\title{
The caregiver perspective: an assistive AAL platform
}

\author{
Angelo Costa ${ }^{1, *}$, Paula Magalhães ${ }^{2,3}$, José Ferreira-Alves ${ }^{2}$, Tito Peixoto ${ }^{4}$, \\ Ricardo Simoes ${ }^{3,5,6}$, Paulo Novais ${ }^{1}$ \\ ${ }^{1}$ CCTC-Computer Science and Technology Center, University of Minho, Braga, Portugal \\ \{acosta, pjon\}@di.uminho.pt \\ ${ }^{2}$ School of Psychology, University of Minho, 4710-057 Braga, Portugal \\ \{pmagalhaes, alves\}@psi.uminho.pt \\ ${ }^{3}$ Institute for Polymers and Composites IPC/I3N, University of Minho, Guimarães, Portugal \\ rsimoes@dep.uminho.pt \\ ${ }^{4}$ Santa Casa da Misericórdia de Vieira do Minho, Portugal \\ ${ }^{5}$ Life and Health Sciences Research Institute (ICVS), School of Health Sciences, University of \\ Minho, Campus de Gualtar, 4710-057 Braga, Portugal \\ ${ }^{6}$ Polytechnic Institute of Cavado and Ave, Campus do IPCA, 4750-810 Barcelos, Portugal \\ rsimoeseipca.pt
}

\begin{abstract}
The Ambient Assisted Living area has spawned several projects that aim to help the user on his/her daily activities. The AAL4ALL (ambient assisted living for all) project aims to develop a unified ecosystem using fully compatible devices and services. The UserAccess platform is part of the AAL4ALL and has as a goal to provide assistance to a type of actor that is commonly forgotten in the Ambient Assisted Living area, the caregiver. This paper presents the architecture, implementation, and interfaces, along with a brief analysis of caregiver's needs and work related issues.
\end{abstract}

Keywords: Ambient Assisted Living; Ambient Intelligence; Intelligent Environments; AAL4ALL; e-Health; Active Ageing; Artificial Intelligence

\section{Introduction}

In a not so distant future, it is expected a complete inverse of the population's pyramid [1]. Specifically, estimates are that by 2050 people aged 65 or older will represent about $21 \%$ of the world's population, and $25 \%$ in places such as the European Union $[2,3]$. This fact creates profound challenges to each nation worldwide: who will take care of the people who need assistance in one or more activities of daily living, and, importantly, who will pay for the solutions?

The challenge itself suggests that one cannot solely rely on people as a solution, as people in working-age will become a scarce asset, and thus an expensive one. Therefore, the act of caring itself needs to be reinvented. This reinvention can be achieved to some degree with the help of a technological approach, although some challenges of the caring process already have technological solutions [4]. The key idea is to create technological solutions that allow to postpone, or completely eliminate the need for full 
time caring that many individuals request and require nowadays. If technological solutions could lessen the physical contact-time of the caregiver without diminishing the individualized and effective care, it would certainly have a great impact on the lives of caregivers and care receivers. Another central idea is to allow for a complete monitoring of the elderly through digital means, and digital contact between the old person and the caregiver, that is, without the need of commuting.

a)
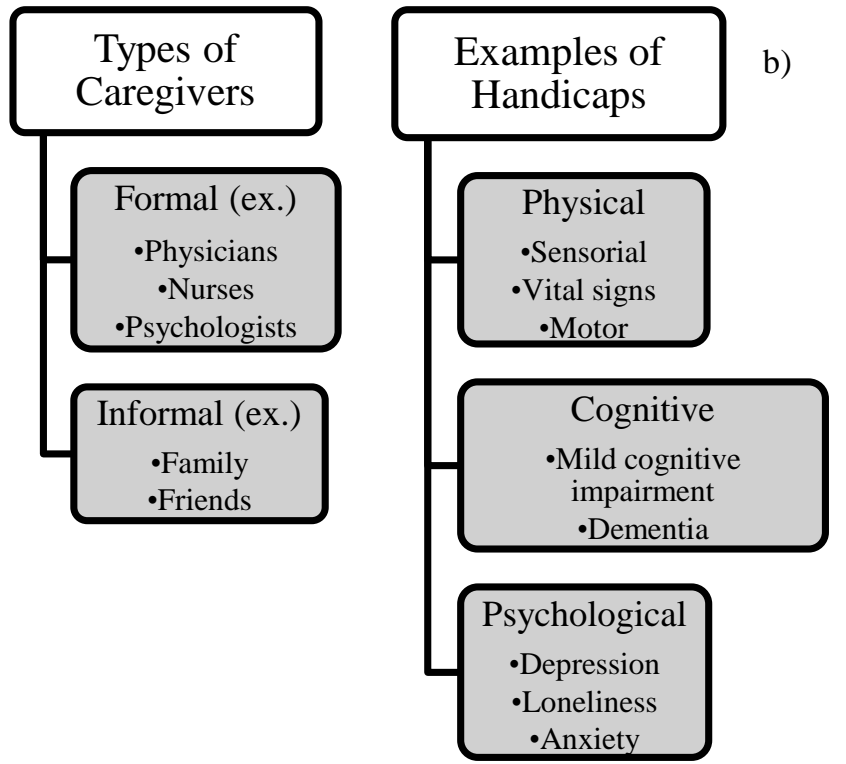

Fig. 1. Types of caregivers (a) and Examples of Handicaps of the care receivers (b).

Caring, however, can assume many forms, and be performed by many different actors; it can occur in a variety of settings, and be more or less formal. Specifically, in a primary care setting, or hospital setting, we can find different formal carers: physicians, nurses, psychologists, medical auxiliaries, among others (Figure 1a). Each of these professionals will have different needs regarding technological solutions, and different expectations about what technology can do for them. Additionally, the care receiver can present a variety of handicaps that pose different challenges to the caregivers and ultimately to those developing technological solutions (Figure 1b). Because of this, the technology that will be developed has to be flexible to adapt to the different profiles of carers, and affordable and intuitive so that it can reach the maximum number of users. Considering the diversity of people who provide care, as well as the diversity of possible settings of intervention, we consider that, overall, the pattern of usage of the new technologies will differ between informal and formal carers, and also within the variety of formal carers. Finally, we consider that communication is a key requirement that needs to be addressed by future solutions.

This document is constituted by the following sections: section 2 presents the caregiver's perspective of an AAL platform; section 3 presents the AAL4ALL project, its 
architecture and structure; section 4 shows the UserAccess project; and finally, section 5 presents the conclusions and the future work.

\section{Caregiver's Perspective}

To be a caregiver of a family member is a role that has plenty potential for facing multiple demands that disrupt the individual's wellbeing [5]. On the one hand, there are challenges that every individual faces in their lifetime to maintain wellbeing and health: self-care, nurture of relationships with family and friends, keep engagement with the community, practice prevention, among others. On the other hand, there are demands associated with and specific to the caregiver's role: being effective in the care provided; receive support and adequate supervision, mainly when the caring tasks are new and challenging; being available to the person being cared for; and, conciliate own needs with the needs of the care-receiver, among others [6].

We can conceive that elderly's informal caregivers could be particularly concerned about the parent or relative's wellbeing or location, if has taken the prescription, whether he/she is feeling lonely, or in urgent need. Informal caregivers could also be concerned whether the house's doors and windows are open, or if the oven and lights are on. Technology's role could be of alleviating some of these demands and concerns raised by the act of caring. This could be achieved by, for instance, facilitating the communication between individuals; monitoring the elderly's house conditions; or creating notifications about the elderly's activities and appointments. For instance, an agenda shared with the caregiver, a list of to-do tasks, or a list of medicine that the elderly has to take, which could be ticked when performed, among other information that the caregiver could remotely have access to. Having an easy access to information related to the elderly could drastically reduce the amount of stress and overload associated with caring. Caregiver's overload can result from the actual number of hours devoted to caring - which could be reduced by technological solutions - or from the special characteristics of the person being cared. Additionally, overload can manifest in several ways, such as: difficulties in sleeping, changes in mood, or eating patterns; struggling to balance all aspects of his/ her life; through physical pain and complaints, or exhaustion. These symptoms clearly lessen the ability caregiver's have to look after others. Therefore, to develop a set of solutions that have the potential to alleviate the stress associated with caring would greatly reduce the strain caregiver's face, improve their quality of live, and ultimately improve the quality of care provided.

Regarding formal caregivers, one can conceive that they have different needs and expectations regarding the role that new technologies can assume in their profession when compared with informal caregivers, but also when comparing different professionals. Physicians and nurses, for instance, could greatly benefit of monitoring of vital signs, teleappointments, and telediagnostics technologies, whereas medical auxiliaries will hardly benefit with these technologies. However, medical auxiliaries could greatly benefit with systems that allow them to monitor the elderlies' performance of the activities of daily living, or to monitor the environment in which the elderly is living in. 
A practical example could be that of a medical auxiliary in a nursing home. In Portuguese nursing homes, this person is responsible for several aspects of the elderly's wellbeing, namely, bathing, checking and caring after the skin condition, accompanying the elderly to the lunch room, among others. All these tasks should be registered and, in case there is a problem, communicate it to the person responsible for addressing that particular issue. For instance, if the elderly is not eating well, the auxiliary should record that information and notify the nursing home's nutritionist. However, there are several obstacles to successfully accomplish these goals. The process of recording is complex and takes time that should be devoted to taking care of the patient. Moreover, there is not a reliable vehicle to communicate the information about and the needs of the elderly to the appropriate professional. That is, sometimes the communication between the auxiliary and the other professionals is not effective and is prone to errors as does not rely in a notifications system. Another obstacle relates to the fact that medical auxiliaries tend to be less educated than other professionals. Finally, it is important to understand that although an individual completes every required task, it does not necessarily imply that the final result is the expected or desired one.

All considered, there is the need for platforms promoting and facilitating communication between the different actors involved in caring. The solutions will need to be simple and of easy interaction so that users from all educational backgrounds can learn how to use them effectively. The solution should allow for the creation of notifications, and ideally this system should be able to differentiate between different degrees of urgency of the different kinds of notification. A system with these characteristics would reduce stress and anxiety inherent to caregiving, and would increase the caregiver's feelings of control, self-worth and accomplishment in his/her work, and within the colleagues. This would not only increase satisfaction in the workplace, but also prevent potential negligence and abuse.

\section{AAL4ALL}

The AAL4ALL project is a Portuguese consortium whose goal is to provide its users with smart care. Being composed of hardware and software companies and universities this project is developing technological geriatric solutions. Some solutions are directed to the elderly and disabled people, which will help on their daily tasks, whereas other solutions focus on the informal and formal caregivers. This approach leads to an innovative AAL ecosystem, that not only focus on the elderly and disabled users, but also in those directly connected to them, increasing what is known as the users sphere and incorporating all actors directly and indirectly involved .

In terms of technological advances, AAL4ALL aims for two main features: integration and certification. As demonstrated in [7] there are a great number of projects that do not provide enough resources or are simply uninterested in integrating with other solutions, which can be a major problem. Specifically, in [8] it is clearly demonstrated that there are many projects that are overlapping their efforts to provide solutions in the same ambit, whereas other domains, such as the caregiver assistance, remain poor in technological platforms. Most of the current projects direct their efforts to the final user, 
leading to an unbalanced distribution of the AAL ecosystem distribution. Additionally, this becomes a problem to the user, as typically a combination of devices and services must be used and, without integration, the full potential of each product is not achieved. In addition, features such as machine learning and artificial intelligence cannot be implemented due to the lack of combined information and individual data processing [9].

The certification is crucial to the correct deployment of the devices and services to the market. As evidenced in [10-12], projects developed without a plan to a possible commercialization end with obsolete products, belonging only to the academic realm. Importantly, there is the need for certification as most of the AAL solutions are imminently (and some really are) medical products. Even software can be considered a medical product, due to the use of private and medical information, by being able to control medical devices, and to generate reports that will be used by medical personnel.

The AAL4ALL solutions range from body and home sensors to virtual actors that interact with the users, enlarging the monitoring and actuation sphere that usually AAL projects are bounded to. This tactic complies with the integration and certification goals, by keeping all solutions under the same umbrella and providing them with modular abilities. Being a complete ecosystem means that all people involved in the task of aiding the care-receiver, such as relatives and professionals, must be considered when developing solutions that ease their interaction with the care-receiver. That is the case of the UserAccess platform, a solution with the goal of integrating all users and connect them with each other.

\section{UserAccess}

The UserAccess platform aims to offer an assistive platform to the formal and informal caregiver [13, 14]. It provides automatized warnings, user environment monitoring, and events managing. Currently, the work of the formal caregiver is stressful and requires long hours. This means a decline in terms of efficiency and attention, and the UserAccess platform aims to alleviate the workload and allow more users to be monitored. Several studies [15-17] relate long hours, exigency and attention to a decrease in attention and increasing stress. Therefore, the user cannot benefit from a good service, and most importantly, in critical situations the response of the caregivers may not be the appropriate and put the care-receiver in great risk. Hence, the UserAccess provides a technological solution to create a more harmonious environment and ultimately provide additional security to the care-receiver and the caregiver.

The UserAccess is built to be used by every AAL4ALL user (caregiver and carereceiver), but tuned to the caregivers. For instance, the informal caregiver, including relatives and friends, can access to comprehensible information about the user and schedule events with him/her. In terms of architecture, the UserAccess platform is currently available in mobile (resorting to Android operating system) and web page formats. It is structured by a server that connects to the AAL4ALL platform, sustaining a modular structure based in multi-agent systems. Figure 2 shows the overview of the architecture, in which the AAL4ALL and the UserAccess platforms are highlighted. 
In terms of operation, the UserAccess receives high-level information, such as "user exited home" or "lights are on", through the UserAccess MQ Node, which saves information in a queue and performs a verification of the incoming data, checking the validity and restructuring it according to the internal communication method. Furthermore, the messages form the other users and external services arrive and go through this module, serving as the gateway to all information entering and exiting.

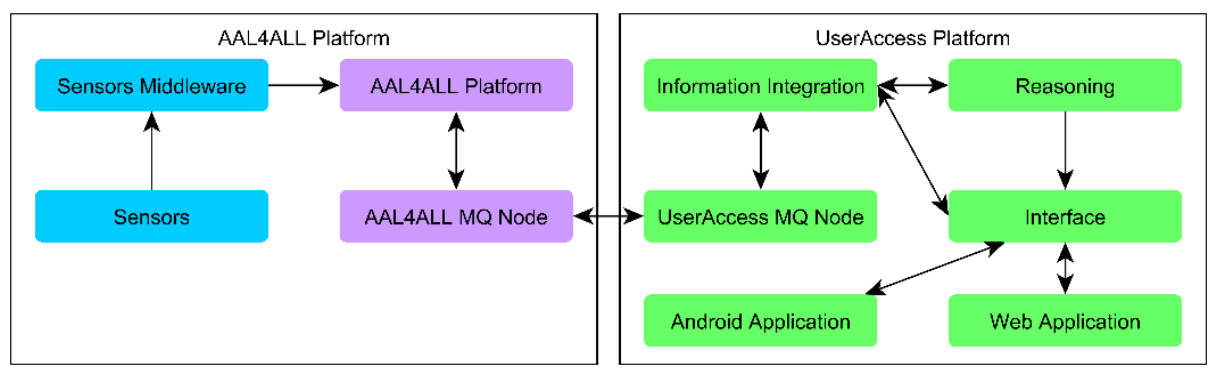

Fig. 2. UserAccess platform overview

The information is sent to the Information integration, being sorted according to the context it possesses. For instance, whether it is a warning coming from a sensor or a calendar update. The information can then be directly sent to the interfaces or further processed in the Reasoning module. The Reasoning module is the most complex module of the platform, being in fact composed of several logical agents. These agents are responsible for the complex processing of information, for instance, if an event is received, it must fetch all calendars, verify the collisions, and, if possible, schedule or find another suitable time frame. The use of several agents was adopted to benefit from the heterogeneity they present, easy upgradability, and communication flow flexibility that makes the addition of new agents easy to perform and integrate with the rest of the agents. Finally, a set of actions is devised and transformed into information or notifications to send to the caregiver mobile platform.

The interfaces are of the uttermost importance, being the tangible element to all users. Thus, it is imperative that interfaces appeal and can conjugate with the users. Figure 3 displays the interfaces developed. Currently, they are able to display the most important messages and the overall information, as well as receive events and display them directly on the interface. Furthermore, an SOS and shortcuts have been placed to ease the task of calling to predefined people or services.

The calendar module is one of the most important features of the platform. As stated before, freeing the caregiver of constant attention is crucial, and, in our perspective, the calendar can serve to micromanage some aspects of each care-receiver's life. The UserAccess android application is built to manage several care-receiver's that the caregiver is responsible for, as it can be seen in Figure 3 a), and, resorting to the calendar, to monitor each aspect of the care-receiver's life (even warnings). 


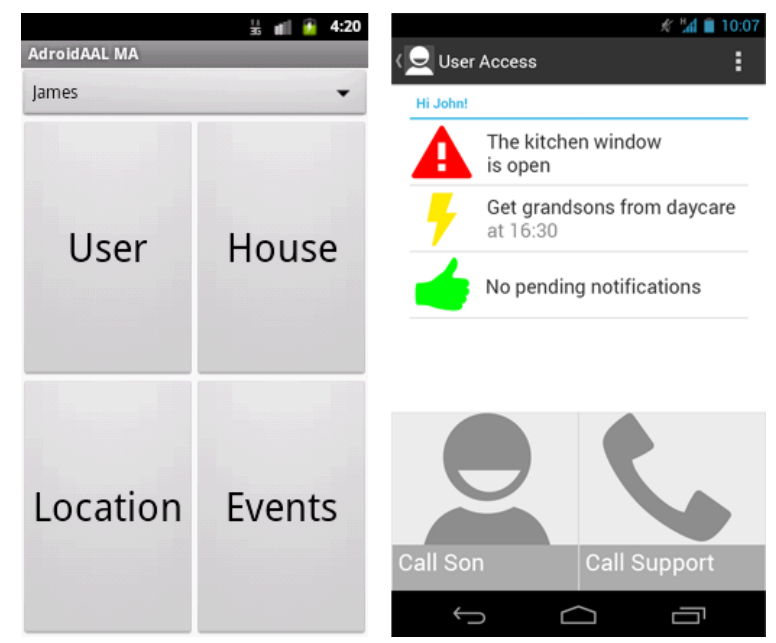

Fig. 3. a) The Android application home interface, b) Alpha stage caregiver interface (warnings and activities).

\section{Conclusions and Future Work}

In this paper was presented the UserAccess project. The AAL4ALL project allows the heavy data processing to be done on the cloud, where the UserAccess is expected to work. The UserAccess is currently focusing on three features: the agenda management, automatic profiling, and user interfaces. The agenda management is currently the core of the platform, being the profiling essential to determine the preferred activities and peers of the users. The users' interfaces are being rebuilt to better accommodate information and improve in style.

Currently, we can state that the UserAccess is close to completion, most of it having already been implemented. The Reasoning module is undergoing several developments and is expected to be fully operational during the current year.

\section{Acknowledgments}

Project "AAL4ALL", co-financed by the European Community Fund FEDER, through COMPETE - Programa Operacional Factores de Competitividade (POFC). Foundation for Science and Technology (FCT), Lisbon, Portugal, through Project PEstC/CTM/LA0025/2013 and the project PEst-OE/EEI/UI0752/2014.

Project CAMCoF - Context-aware Multimodal Communication Framework funded by ERDF -European Regional Development Fund through the COMPETE Programme (operational programme for competitiveness) and by National Funds through the FCT - Fundação para a Ciência e a Tecnologia (Portuguese Foundation for Science and Technology) within project FCOMP-01-0124-FEDER-028980. 


\section{References}

1. United Nations: Population estimates and projections section. (2012).

2. United Nations, Department of Economic and Social Affairs, P.D.: World Population Ageing 2013. (2013).

3. Grauel, J., Spellerberg, A.: Attitudes and requirements of elderly people towards assisted living solutions. In: Mühlhäuser, M., Ferscha, A., and Aitenbichler, E. (eds.) Constructing Ambient Intelligence. pp. 197-206. Springer Berlin Heidelberg, Berlin, Heidelberg (2008).

4. Rashidi, P. \& Mihailidis, A. A survey on ambient-assisted living tools for older adults. IEEE Journal of Biomedical and Health Informatics, 17, 579-590 (2013).

5. Hirst, M. Carer distress: a prospective, population-based study. Social Science \& Medicine, 61, 697-708 (2005).

6. Nolan, M. R. \& Grant, G.. Addressing the needs of informal carers: a neglected area of nursing practice. Journal of Advanced Nursing, 14, 950-961 (1989).

7. Norgall, T., Wichert, R.: Towards Interoperability and Integration of Personal Health AAL Ecosystems. Studies in health technology and informatics. pp. 272-82. IOS Press (2012).

8. Antonino, P.O., Schneider, D., Hofmann, C., Nakagawa, E.Y.: Evaluation of AAL platforms according to architecture-based quality attributes. Ambient Intelligence. pp. 264-274 (2011).

9. Fagerberg, G., Kung, A., Wichert, R., Tazari, M.R., Jean-Bart, B., Bauer, G., Zimmermann, G., Furfari, F., Potort, F., Chessa, S., Hellenschmidt, M., Gorman, J., Alexandersson, J., Bund, J., Carrasco, E., Epelde, G., Klima, M., Urdaneta, E., Vanderheiden, G., Zinnikus, I.: Platforms for AAL applications. Smart Sensing and Context. pp. 177-201. Springer Berlin Heidelberg (2010).

10. Kung, A., Jean-Bart, B.: Making AAL Platforms a Reality. Ambient Intelligence. pp. 187196. Springer Berlin Heidelberg, Berlin, Heidelberg (2010).

11. Hanke, S., Mayer, C., Hoeftberger, O., Boos, H., Wichert, R., Tazari, M.-R., Wolf, P., Furfari, F.: universAAL - An Open and Consolidated AAL Platform. In: Wichert, R. and Eberhardt, B. (eds.) Ambient Assited Living 4 Deutscher AALKongress. pp. 127-140. Springer (2011).

12. Walderhaug, S., Mikalsen, M., Salvi, D., Svagård, I., Ausen, D., Kofod-Petersen, A.: Towards quality assurance of AAL services. Stud. Health Technol. Inform. 177, 296-303 (2012).

13. Costa, A., Novais, P., Simoes, R.: A caregiver support platform within the scope of an ambient assisted living ecosystem. Sensors (Basel). 14, 5654-5676 (2014).

14. Costa, A., Gama, O., Novais, P., Simoes, R.: A Different Approach in an AAL Ecosystem: A Mobile Assistant for the Caregiver. Highlights of Practical Applications of Heterogeneous Multi-Agent Systems. The PAAMS Collection. pp. 101-110. Springer International Publishing, Salamanca, Spain (2014).

15. Robinson-Whelen, S., Tada, Y., MacCallum, R.C., McGuire, L., Kiecolt-Glaser, J.K.: Longterm caregiving: What happens when it ends? J. Abnorm. Psychol. 110, 573-584 (2001).

16. Schulz, R., Mendelsohn, A.B., Haley, W.E., Mahoney, D., Allen, R.S., Zhang, S.,Thompson, L., Belle, S.H.: End-of-life care and the effects of bereavement on family caregivers of persons with dementia. N. Engl. J. Med. 349, 1936-42 (2003).

17. Sherwood, P.R., Donovan, H.S., Given, C.W., Lu, X., Given, B.A., Hricik, A., Bradley, S.: Predictors of employment and lost hours from work in cancer caregivers. Psychooncology. 17, 598-605 (2008). 